\title{
Recovering the lung transplant service after COVID-19 - experience at the Freeman Hospital, Newcastle
}

\author{
Authors: Falak Umair, ${ }^{A}$ Muhammad Waseem Athar, ${ }^{\mathrm{A}}$ Arun Nair, ${ }^{\mathrm{A}}$ James Lordan, ${ }^{\mathrm{A}}$ Andrew Fisher $^{\mathrm{A}}$ and \\ Gerard Meachery ${ }^{\mathrm{A}}$
}

\section{Background}

COVID-19 caused a significant impact on organ transplantation. In the UK, during the peak months the number of deceased donors and transplants fell by $66 \%$ and $68 \%$ respectively compared with last year. ${ }^{1}$ The lung transplant service at the Freeman Hospital, Newcastle covers a wide geographical area including Northern Ireland, Scotland and northern England. The lung transplant service was suspended in the midst of COVID-19. Transplant staff were redeployed to help manage the COVID-19 response. Recovering the service was difficult due to the limited number of donated lungs and extreme pressure on our services. Ensuring patient safety in this high-risk group was crucial. $^{2}$

\section{Methods}

A significant backlog of patients were awaiting inpatient assessment to decide their suitability for lung transplant. As inpatient investigative capacity was limited, stringent rules were enforced to ensure that all necessary investigations and data were available before assessment. Transplant education, psychological evaluation and social worker reviews were performed electronically. This reduced the inpatient stay from 4 to 2 days.

To restrict patient footfall, ${ }^{3}$ the entire inpatient journey was mapped out. All patients admitted were shielding and tested locally for COVID-19. They were re-tested on admission. Ward reconfiguration and strict allocation for only lung transplant assessments was enforced to ensure safe distancing. Appropriate safe spaces were designated for physiotherapy assessment. Departmental tests were arranged to ensure that they were performed at specified time allocations with minimal contact. Strict infection control and personal protective equipment (PPE) protocols were enforced.

A combination of telephone and limited face-to-face clinics were facilitated for pre-transplant patients. Strict distancing, appropriate PPE and limitation on numbers were ensured during these visits.

Teleconferencing was utilised for the multidisciplinary team (MDT) meeting to allow social distancing and attendance of all. The MDT outcome was communicated via telephone or video

Author: ${ }^{\text {A } F r e e m a n ~ H o s p i t a l, ~ N e w c a s t l e ~ u p o n ~ T y n e, ~ U K ~}$ conference. Surgical and anaesthetic consent was taken via video conference.

All patients on the active transplant waiting list were contacted about risks and changes due to COVID-19. Patients admitted for surgery were screened for symptoms and contact with COVID-19 patients. A rapid COVID-19 test was used on admission. Patients were re-consented and risk of COVID-19 was discussed again prior to surgery.

\section{Results}

Patients (April-September 2020):

$>$ Number of new referrals 75

$>$ Assessed in clinic (new plus old referrals) 115

$>$ Admitted for in-patient assessment 25

$>$ Lung transplants performed 10

$>$ COVID-19 in above patients 0

\section{Conclusions}

COVID-19 resulted in complete reconfiguration of our service. Pathways were streamlined and technology updated to enable a safe recovery of the programme. Patient and staff safety to reduce the risk of COVID-19 was central to planning this reconfiguration.

\section{Conflicts of interest}

None declared.

\section{References}

1 Manara AR, Mumford L, Callaghan CJ, Ravanan R, Gardiner D. Donation and transplantation activity in the UK during the COVID-19 lockdown. Lancet 2020;396:465-6.

2 NHS Blood and Transplant. Re-opening of transplant programmes: issues for consideration. NHS, 2020. http://nhsbtdbe.blob.core. windows.net/umbraco-assets-corp/18627/pol296.pdf [Accessed 02 December 2020].

3 International Society of Heart and Lung Transplantation. Guidance from the International Society of Heart and Lung Transplantation regarding the SARS CoV-2 pandemic. ISHLT, 2020. https:// ishlt.org/ishlt/media/documents/SARS-CoV-2_-Guidance-forCardiothoracic-Transplant-and-VAD-centers.pdf [Accessed 02 December 2020]. 